OPEN ACCESS

Edited by:

Lena Elisabeth Friberg,

Uppsala University, Sweden

Reviewed by:

Bernd Meibohm,

University of Tennessee Health Science Center (UTHSC),

United States

Sebastian G. Wicha,

University of Hamburg, Germany

*Correspondence:

Shashikant Srivastava

Shashi.kant@uthct.edu

${ }^{+}$These authors have contributed equally to this work

Specialty section: This article was submitted to Translational Pharmacology, a section of the journal Frontiers in Pharmacology

Received: 22 December 2020 Accepted: 04 March 2021

Published: 15 April 2021

Citation:

Boorgula GD, Jakkula LUMR, Gumbo $T$, Jung $B$ and Srivastava $S$ (2021) Comparison of Rifamycins for Efficacy Against Mycobacterium avium Complex and Resistance Emergence in the Hollow Fiber Model System.

Front. Pharmacol. 12:645264. doi: 10.3389/fphar.2021.645264

\section{Comparison of Rifamycins for Efficacy Against Mycobacterium avium Complex and Resistance Emergence in the Hollow Fiber Model System}

\author{
Gunavanthi D. Boorgula ${ }^{1+}$, Laxmi U. M. R. Jakkula ${ }^{1+}$, Tawanda Gumbo ${ }^{2}$, Bockgie Jung ${ }^{1}$ and \\ Shashikant Srivastava ${ }^{1,3 *}$ \\ ${ }^{1}$ Department of Pulmonary Immunology, University of Texas Health Science Center at Tyler, Tyler, TX, United States, ${ }^{2}$ Quantitative \\ Preclinical and Clinical Sciences Department, Praedicare Inc., Dallas, TX. United States, ${ }^{3}$ Department of Immunology, UT \\ Southwestern Medical Center, Dallas, TX, United States
}

Rifamycins are integral part of the combination regimen for treatment of pulmonary Mycobacterium avium-complex [MAC] infection, but different practitioners prefer different rifamycins. The objective of the study was to compare microbial kill and resistance emergence of rifamycins using principles of pharmacokinetics/pharmacodynamics. First, we identified rifamycin MICs in 20 MAC isolates from patients followed by concentrationresponse studies in test-tubes. Next, we examined efficacy and resistance suppression of three doses of each rifamycin in the hollow fiber system model of pulmonary MAC [HFS$\mathrm{MAC}$ ], mimicking human like concentration-time profile of the drugs. HFS-MAC units were repetitively sampled for total and drug-resistant MAC burden and for drug concentration measurements. Inhibitory sigmoid $\mathrm{E}_{\text {max }}$ model, linear regression, and analysis of variance was used for data analysis. For rifabutin $90 \%$ of isolates had $\mathrm{MIC} \leq 0.125 \mathrm{mg} / \mathrm{L}$ while for both rifampin and rifapentine this was $\leq 2.0 \mathrm{mg} / \mathrm{L}$. There was no statistically significant difference $(p>0.05)$ in maximal kill and effective concentration mediating $50 \%$ of the bacterial kill among three rifamycins in the static concentration experiment. In the HFS-MAC, the bactericidal kill (day 0-4) for rifampin was 0.89 (95\% Confidence Interval (Cl): 0.43-1.35), for rifapentine was 1.05 (95\% Cl: 0.08-1.23), and for rifabutin was 0.92 (95\% Cl: 0.61-1.24) $\log _{10} \mathrm{CFU} / \mathrm{ml}$, respectively. Rifamycins monotherapy failed after just 4-days of treatment and entire MAC population was drug resistant on day 26 of the study. There was no dose dependent difference in MAC kill or resistance suppression among the three rifamycins tested in the HFS-MAC. Therefore, replacing one rifamycin, due to emergence of drugresistance, with other may not be beneficial in clinical setting.

Keywords: rifampin, rifapentine, rifabutin, pharmacokinetics/pharmacodynamics, resistance

\section{INTRODUCTION}

Nontuberculous mycobacteria (NTM), especially species of the Mycobacterium avium complex (MAC), are among the most difficult to treat pulmonary infection (Cowman et al., 2019). A recent retrospective study showed that between 2008 to 2015, the annual NTM lung disease incidence increased from 3.13 to 4.73 per 100,000 person, and the annual prevalence changed from 6.78 to 11.7 (Winthrop et al., 2020). During the same period, the prevalence among the women increased 
from 9.63 to 16.7 per 100,000 , while among those 65 years or older it increased from 30.27 to 47.48 per 100,000 (Winthrop et al., 2020). The current American Thoracic Society [ATS] guidelines recommend a macrolide-ethambutol-rifamycin combination therapy for treatment of pulmonary MAC (Griffith, 2007). However, as per a recent meta-analyses, despite 18-23 months long therapy duration, the sustained sputum culture conversion rates at the end of therapy, a marker of cure, were only $54 \%$ (Pasipanodya et al., 2017). Just as clinicians use different macrolides (azithromycin or clarithromycin], they also use different rifamycins [rifampin or rifabutin). It is unclear which rifamycin is better; moreover, how rifapentine would fare in treatment of pulmonary MAC is currently unclear. We compared the three rifamycins head-to-head using pharmacokinetics/ pharmacodynamic (PK/PD) study design.

We have developed a pre-clinical hollow fiber system model of pulmonary MAC (HFS-MAC), that can mimic the human like PK of the drugs (Deshpande et al., 2010a; Deshpande et al., 2010b; Deshpande and Gumbo, 2011; Deshpande et al., 2016b; Deshpande et al., 2017a; Deshpande et al., 2017b; Deshpande et al., 2017c; Deshpande et al., 2017d; Srivastava et al., 2017a; Srivastava et al., 2017b). We have used the HFS-MAC for PK/PD studies with azithromycin, ethambutol, moxifloxacin, thioridazine, linezolid, tedizolid, ceftazidime-avibactam, minocycline, and tigecycline (Deshpande et al., 2010a; Deshpande et al., 2010b; Deshpande et al., 2016a; Deshpande et al., 2016b; Deshpande et al., 2017a; Deshpande et al., 2017b; Deshpande et al., 2017d). In the present study, we utilized the same model system for rifamycins $\mathrm{PK} / \mathrm{PD}$ that allowed us to compare how this class of drugs perform relative to other drugs used to treat pulmonary MAC infection.

\section{MATERIALS AND METHODS}

\section{Bacteria, Media, and Other Supplies}

The study protocol was approved by the institutional infectious organism research review committee. All experiments were performed in the BSL2 laboratory. Prior to each experiment the stock culture of MAC (American Type Culture Collection, ATCC\#700898 and 20 clinical isolates provided by Dr Barbara Brown-Elliot, Department of Microbiology, University of Texas Health Science Center at Tyler, TX) were grown to log phase growth at $37^{\circ} \mathrm{C}$ in Middlebrook $7 \mathrm{H} 9$ broth supplemented with $10 \%$ oleic acid-albumin-dextrose-catalase (OADC) [herein, termed "broth"] under shaking condition. Hollow fiber cartridges were purchased from FiberCell (Frederick, MD). Drugs were purchased from Sigma Aldrich. Drugs were first dissolved in $100 \%$ dimethyl sulfoxide [DMSO] followed by dilution in $25 \%$ DMSO to achieve the desired concentration to use in the experiments. The final concentration of DMSO was kept at $<1 \%$, having no effect on MAC growth.

\section{Determination of Minimum Inhibitory Concentration}

MIC of rifampin, rifapentine, and rifabutin was determined using the broth micro-dilution method (CLSI, 2018). Briefly, bacteria (standard laboratory strain, ATCC\#700898, as well as 20 clinical isolates) were grown to log-phase growth in broth to an optical density of $0.07-0.08$ at a wavelength of $600 \mathrm{~nm}$, corresponding to McFarland standard 0.5 (McFarland, 1907). The cultures were then diluted 100-fold in broth to achieve a bacterial density of $\sim 10^{5} \mathrm{CFU} /$ $\mathrm{ml}$. Next, $180 \mu \mathrm{L}$ of the cultures were added to each well of 96-well plates, pre-filled with $20 \mu \mathrm{L}$ of the drugs at $10 \mathrm{x}$ concentration (10fold dilution). The final drug concentrations were 0 (non-treated), $0.03125,0.0625,0.125,0.25,0.5,1.0$, and $2 \mathrm{mg} / \mathrm{L}$. The plates were sealed in plastic bags and cultures were incubated at $37^{\circ} \mathrm{C}$ under for 7 days. On day 7, the plates were read using an inverted mirror and MIC was defined as the lowest drug concentration that completely inhibited visible microbial growth in the wells. The experiment was performed twice with two replicates for each drug concentration.

\section{Rifamycins Concentration-Response Study in Test-Tubes}

The experiment was performed only with the standard laboratory strain of MAC. The method for inoculum preparation was same as described above. The concentrations of rifampin, rifapentine, and rifabutin were 0 (non-treated), 0.03125, 0.0625, 0.125, 0.25, $0.5,1.0$, and $4 \mathrm{mg} / \mathrm{L}$. The experiment was performed in triplicate with a total volume of $5 \mathrm{ml}$ in $15 \mathrm{ml}$ screw caped tubes. MAC cultures with each drug concentration were co-incubated for 7 days, followed by washing twice in normal saline to remove carry over drug, then 10-fold serial dilution and finally culture on Middlebrook 7H10 agar supplemented with 10\% OADC (herein termed "agar"). The cultures were incubated at $37^{\circ} \mathrm{C}$ for 10-14 days before colony forming units (CFU) were recorded.

\section{Comparison of Rifamycins Using the Hollow Fiber Model System}

The detailed description of the HFS-MAC has been published elsewhere (Deshpande et al., 2010a; Deshpande et al., 2010b; Srivastava and Gumbo, 2011; Srivastava et al., 2017a). To summarize, the central compartment of the HFS-MAC receives the drugs administered via computer-controlled syringe pump. The drug infusion rate determines the time $\left(\mathrm{T}_{\max }\right)$ to reach the peak concentration $\left(\mathrm{C}_{\max }\right)$ with given human equivalent dose. The continuous infusion of fresh media into the central compartment, at predetermined inflow rate, control the half-life $\left(t_{1 / 2}\right)$ of the drugs in the HFS-MAC. Next, a set of duet pumps circulate the drug containing media from the central compartment to the peripheral compartment, that house semipermeable hollow fiber membranes. The pore size can be selected in such a way it allows the nutrients and drugs to cross the membrane but keep the bacteria in the peripheral compartment. Finally, a second set of peristaltic masterflex pump removes the waste media from the central compartment. Thus, the HFS-MAC serves as a continuous dilution system where the bacteria always remain in contact with fluctuating drug concentration, Based on the previous publications, we used the free $(f)$ drug concentration and resultant drug exposure of rifampin (Gumbo et al., 2007), rifapentine (Egelund et al., 2014), and rifabutin (Blaschke and Skinner, 1996; No_Author_Listed (2014). Mycobutin [Rifabutin] 
TABLE 1 | Rifampin, rifapentine, and rifabutin drug exposure achieved in the HFS-MAC.

\begin{tabular}{|c|c|c|c|c|c|}
\hline Regimen & Drug & $f C_{\max }(\mathrm{mg} / \mathrm{L})$ & fAUC $_{0-24}\left(\mathrm{mg}^{\star} \mathrm{hr} / \mathrm{L}\right)$ & $f C_{\max } / M I C$ & fAUC $_{0-24} / \mathrm{MIC}$ \\
\hline RIF-1 & Rifampin & 0.76 & 5.79 & 26.54 & 192.97 \\
\hline RIF-2 & Rifampin & 2.03 & 19 & 67.63 & 633.33 \\
\hline RIF-3 & Rifampin & 4.12 & 39.84 & 137.2 & 1328 \\
\hline RFP-1 & Rifapentine & 0.15 & 1.38 & 5.1 & 46.1 \\
\hline RFP-2 & Rifapentine & 0.17 & 2.22 & 5.62 & 73.87 \\
\hline RFP-3 & Rifapentine & 1.02 & 13.05 & 33.93 & 435 \\
\hline RFB-1 & Rifabutin & 1.05 & 12.52 & 35 & 417.33 \\
\hline RFB-2 & Rifabutin & 1.98 & 26.91 & 66.07 & 897 \\
\hline RFB-3 & Rifabutin & 3.48 & 48.19 & 115.87 & 1606.33 \\
\hline CTL-1 & Non-treated & - & - & - & - \\
\hline CTL-2 & Non-treated & - & - & - & - \\
\hline CTL-3 & Non-treated & - & - & - & - \\
\hline
\end{tabular}

capsule, USP [Online]. Available: https://www.accessdata.fda.gov/ drugsatfda_docs/label/2014/050689Orig1s018lbl.pdf [Accessed 10/ 20/2020 2020].) to treat the HFS-MAC units. The elsewhere reported protein binding of rifampin, rifapentine, and rifabutin were used to determine the clinically relevant drug exposures to test in the HFS-MAC (Gumbo et al., 2007; Dooley et al., 2012; Naiker et al., 2014). The HFS-MAC units were housed in incubators at $37^{\circ} \mathrm{C}$ under $5 \% \mathrm{CO}_{2}$ and pre-conditioned with broth (where OADC was replaced with $10 \%$ dextrose) for $72 \mathrm{~h}$. We used three different drug exposures, standard as well as high dose, rifamycins as shown in Table 1. (Gumbo et al., 2007; Dooley et al., 2012; Naiker et al., 2014). In the HFS-MAC, we mimicked $3 \mathrm{~h}$ half-life for rifampin and $15 \mathrm{~h}$ for rifapentine and rifabutin. The experiment was performed with one HFS-MAC system per drug exposure and three non-treated controls, thus a total of 12 HFS-MAC units.

Twenty $\mathrm{mL}$ of the log-phase growth cultures of MAC (ATCC\#700898) were inoculated into the peripheral compartment of each HFS-MAC unit. The drugs were infused in the central compartment, over $1 \mathrm{~h}$, using programable syringe pumps. The peripheral compartment of each HFS-MAC unit was sampled on days $0,4,7,14,21$ and 26 . The samples were washed twice with normal saline to remove carry over drug followed by 10-fold serial dilution, as described in detail previously (Deshpande et al., 2010a; Deshpande et al., 2010b; Srivastava et al., 2017a), to estimate the total bacterial burden on Middlebrook 7H10 agar. For enumeration of the drug resistant subpopulation, the same samples were cultured on agar containing $12 \mathrm{x}$ the MIC of each drug. The intent was not to capture the actual change in the MIC, hence single drug concentration was used. For the validation of the concentration-time profile of the drugs, the central compartment of each HFS-MAC unit was sampled on day 26. Thus, these represent steady state drug concentrations. The sampling time-points were as following: pre-dose, then at 1,2 , 3, 612,18 and $23.5 \mathrm{~h}$ post dosing. We used previously described LC-MS/MS based concentration measurement assays, without modification (Gumbo et al., 2007; Deshpande et al., 2017c; Chapagain et al., 2020).

\section{Data Analysis}

The measured drug concentrations were modeled using Phoenix WinNonlin 8.1 (Certara USA, Inc., MO, United States) and

\begin{tabular}{lccc}
\hline TABLE 2 | MIC of M. avium clinical strains against rifamycins. & \\
\hline Strain ID & Rifampin & Rifapentine & Rifabutin \\
\hline 6824 & 0.125 & 0.06 & 0.06 \\
65380 & 0.03 & 0.03 & 0.03 \\
63064 & 0.03 & 0.03 & 0.03 \\
63045 & 0.03 & 0.03 & 0.03 \\
65195 & 0.06 & 0.25 & 0.06 \\
65547 & 0.03 & 0.03 & 0.03 \\
68160 & 0.03 & 0.03 & 0.03 \\
65317 & 0.03 & 0.03 & 0.03 \\
64673 & 0.03 & 0.03 & 0.03 \\
65933 & 0.03 & 1 & 0.03 \\
65406 & 0.03 & 0.03 & 0.03 \\
68246 & 0.03 & 0.03 & 0.03 \\
65485 & 2 & 2 & 0.06 \\
68164 & 2 & 2 & 0.06 \\
68162 & 2 & 2 & 1 \\
65411 & 0.03 & 0.03 & 0.03 \\
65899 & 0.06 & 0.06 & 0.06 \\
65321 & 2 & 2 & 2 \\
63331 & 0.25 & 0.25 & 0.06 \\
65408 & 2 & 2 & 0.125
\end{tabular}

used to calculate the drug exposure in terms of ratio of peak concentration to MIC $\left(\mathrm{C}_{\max } / \mathrm{MIC}\right)$ and $24 \mathrm{~h}$ area under the concentration-time curve to $\mathrm{MIC}\left(\mathrm{AUC}_{0-24} / \mathrm{MIC}\right)$. GraphPad Prism (v 8.0) was used for graphing as well as to determine the relationship between the drug concentration and bacterial burden using the three-parameter $\left(\mathrm{E}_{\mathrm{con}}, \mathrm{E}_{\max }\right.$, EC) inhibitory sigmoid $E_{\max }$ model (i.e., $H$ fixed at 1 ), to perform the linear regression analysis to calculate the kill rate with each drug and dosing regimen, and to perform analysis of variance (ANOVA) to compare the different drug regimens.

\section{RESULTS}

Table 2 and Figure 1A show the rifamycin's MIC distribution in the 20 clinical strains. The $\mathrm{MIC}_{50}$ for all isolates were at the lowest concentrations of drug tested i.e., $0.032 \mathrm{mg} / \mathrm{L}$. The $\mathrm{MIC}_{90}$ of rifabutin was 5-tube dilutions and 16-fold times lower than rifampin and rifapentine. The MIC of the MAC laboratory 

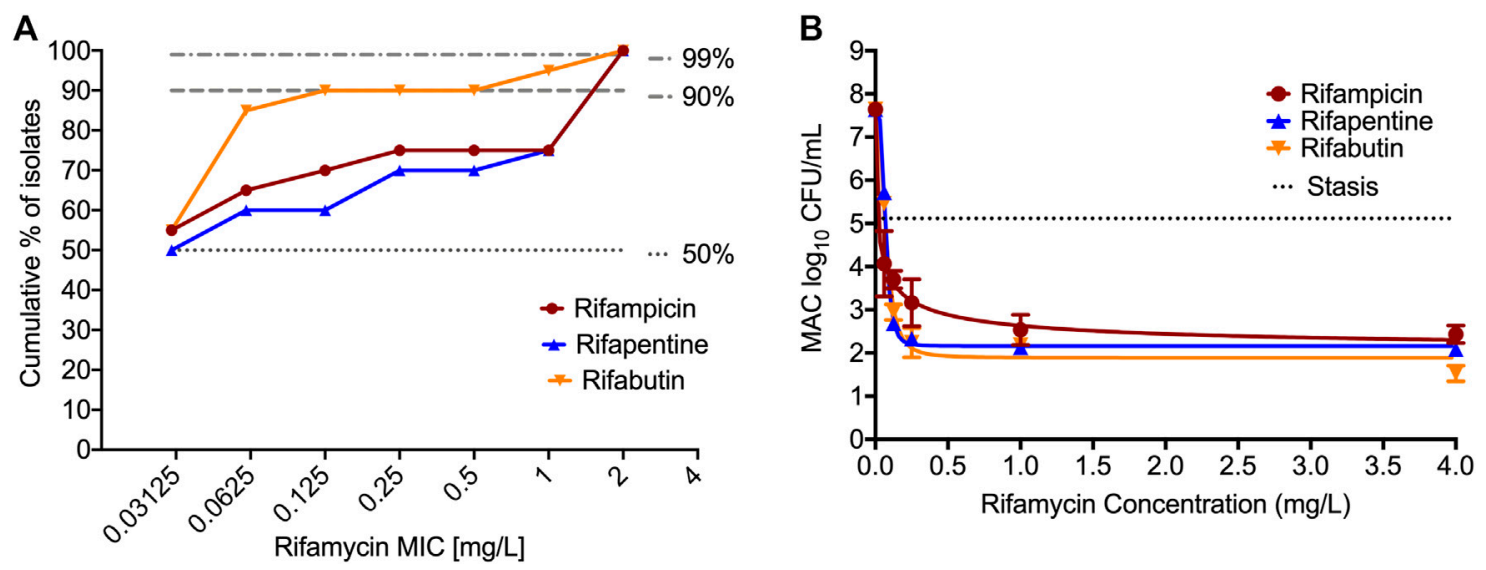

FIGURE 1 | MIC distribution and concentration-response of rifamycins against MIC. (A). MIC distribution of 20 MAC clinical isolates against the three rifamycins. (B). Bacteria was exposed to different static concentrations of the drugs for seven days. The solid line is for model fit, and symbols represent the data point. The kill below stasis [day 0 or inoculum] with rifampin was 2.68 (95\% Confidence Interval (Cl): 1.13 to 4.24) $\log _{10} \mathrm{CFU} / \mathrm{mL}$, for rifapentine was 3.03 (95\% Cl: 2.89 to 3.17 ) log ${ }_{10} \mathrm{CFU} / \mathrm{mL}$, and for rifabutin was 3.59 (95\% Cl: 2.22 to 4.96$) \log _{10} \mathrm{CFU} / \mathrm{mL}$, respectively.

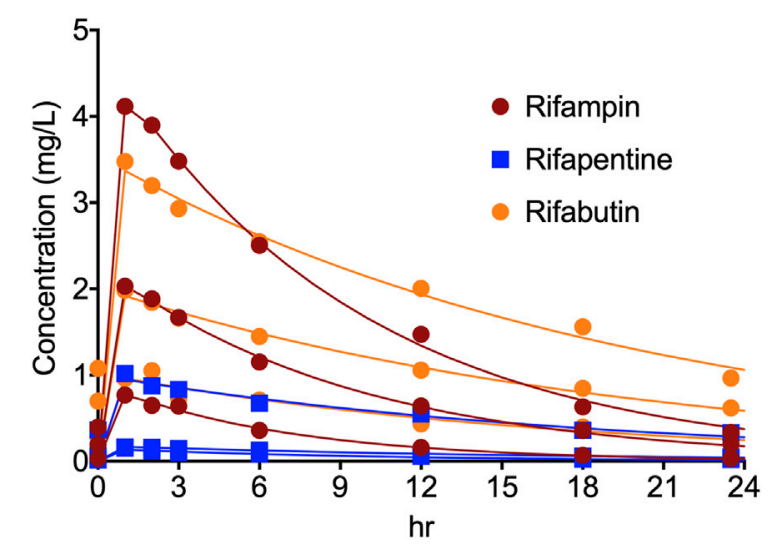

FIGURE 2 | Concentration-time profile of rifampin, rifapentine, and rifabutin in the HFS-MAC. Pharmacokinetics model predicted concentration are shown as solid line and the symbol represent the observed concentrations in the HFS-MAS at each sampling time-point.

strain used for all subsequent PK/PD studies was $0.032 \mathrm{mg} / \mathrm{L}$ for either rifampin, or rifapentine, or rifabutin, in two separate experiments with two replicate each. This isolate was then examined in the static concentration-response studies, with results shown in Figure 1B. The $\mathrm{E}_{\max }$ (maximal kill) and $\mathrm{EC}_{50}$ (concentration mediating $50 \%$ of the $\mathrm{E}_{\max }$ ) of rifampin were 5.674 (95\% Confidence Interval (CI): 4.708-6.641) $\log _{10} \mathrm{CFU} / \mathrm{mL}$ and 0.023 (95\% CI: 0.001 to 0.048$) \mathrm{mg} / \mathrm{L}\left(r^{2}=0.972\right)$; for rifapentine were 5.480 (95\% CI: 5.265 to 5.696$) \log _{10} \mathrm{CFU} / \mathrm{mL}$ and 0.070 (95\% CI: 0.067 to 0.073$) \mathrm{mg} / \mathrm{L}\left(r^{2}=0.998\right)$; and for rifabutin were 5.76 (95\% CI: 5.189 to 6.329$) \log _{10} \mathrm{CFU} / \mathrm{mL}$ and 0.072 (95\% CI: 061 to 0.082$) \mathrm{mg} / \mathrm{L}\left(r^{2}=0.998\right)$, respectively. Therefore, the efficacy $\left(\mathrm{E}_{\max }\right)$ and potency $\left(\mathrm{EC}_{50}\right)$ of the three rifamycins were virtually identical.

Figure 2 show the concentration-time profile of the rifampin, rifapentine, and rifabutin, as achieved in the HFS-MAC. The regression between model predicted vs. measured drug concentrations showed an $r^{2}$ of 0.997 , indicating good model fit. The half-life of the drugs achieved in the HFS-MAC were $5.91 \pm 0.98 \mathrm{~h}, 10.62 \pm 3.03 \mathrm{~h}$, and $13.29 \pm 0.60 \mathrm{~h}$ for rifampin, rifapentine, and rifabutin, respectively. Since there was no protein present in the circulating media, Table 1 summarize the free $(f)$ $\mathrm{C}_{\max }$ and calculated drug exposure $\left(f \mathrm{C}_{\max } / \mathrm{MIC}\right.$ and $f \mathrm{AUC}_{0-24} /$ MIC) of each drug in the HFS-MAC. To put the results in a clinical perspective, rifampin $f C_{\max }$ of $2.03 \mathrm{mg} / \mathrm{L}$ could be interpreted as a clinical dose of $600 \mathrm{mg}$ per day, rifapentine $\mathrm{fC}_{\max }$ of $1.02 \mathrm{mg} / \mathrm{L}$ equivalent to $1200 \mathrm{mg}$ per day, and rifabutin $\mathrm{fC}_{\max }$ of $1.05 \mathrm{mg} / \mathrm{L}$ equivalent to a $900 \mathrm{mg}$ daily dose.

Figures 3A-C show the time kill curves with different rifampin AUC/MIC exposures, and the emergence of resistance with time. At each rifampin monotherapy exposure there was a biphasic effect due to rapid emergence of resistance, with only a slight delay of 4 days at the highest rifampin AUC/ MIC exposure. There was no statistically significant difference between the bacterial burden among the three rifampin exposures and ANOVA showed that the drug exposure could explain only $1.71 \%$ of the variance. Figures $\mathbf{3 A}-\mathbf{C}$ show the change in the rifampin resistant subpopulation that was not statistically different from the total population $(p>0.05)$. Figure 3D and Table 3 show the inhibitory sigmoid $\mathrm{E}_{\max }$ curves and parameters at different rifampin exposures for each sampling timepoint.

Figures $\mathbf{4 A}-\mathbf{C}$ show the time kill curve with different rifapentine AUC/MIC exposures, and emergence of resistance with time. Figure 4D and Table 4 show the inhibitory sigmoid $\mathrm{E}_{\max }$ curves and parameters at different rifampin exposures. Figures $\mathbf{4 A}-\mathbf{C}$ show the change in the MAC bacterial burden in response to the different rifapentine doses over time. The mean bacterial kill with the three rifapentine exposures during the first 4-days was 1.05 (95\% CI: 0.81 to 1.29 ) $\log _{10} \mathrm{CFU} / \mathrm{mL}$ below stasis and the kill rate was $-0.28 \pm 0.03 \log _{10} \mathrm{CFU} / \mathrm{mL} /$ day. However, as shown in Figures $4 \mathrm{~A}-\mathrm{C}$, all three rifapentine exposure failed to control the growth of bacteria in the HFS-MAC, and the entire 

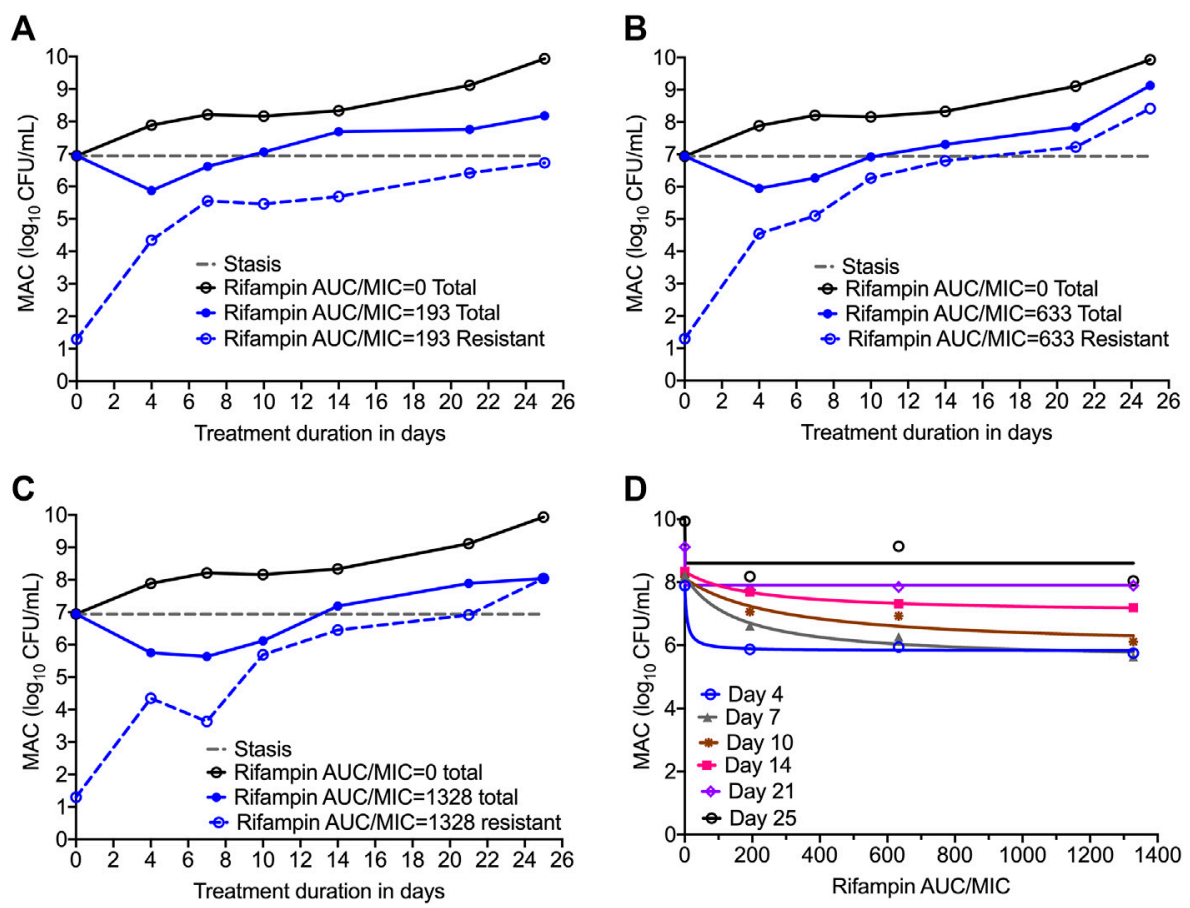

FIGURE 3 | Efficacy of rifampin and resistance emergence in the HFS-MAC. (A-C). All three rifampin exposures showed virtually identical kill of MAC for the first 4days, then failed due to emergence of rifampin resistance in the HFS-MAC. The lowest rifampin exposure AUC/MIC = 193 while failed to control the emergence of drug resistance, had lowest drug-resistant subpopulation by the end of the study. (D). Inhibitory Sigmoid $\mathrm{E}_{\max }$ model to show the relationship between the rifampin exposure and bacterial burden. On day 14 [with highest $r^{2}=0.999$ and lowest Akaike Information Criteria score (Akaike, 1974) = 0.009], EC 50 was calculated as AUC/MIC of 197.3.

TABLE 3 | Rifampin pharmacokinetics/pharmacodynamics indices for microbial kill in the HFS-MAC.

\begin{tabular}{|c|c|c|c|c|c|c|c|}
\hline \multirow[t]{2}{*}{ Study day } & \multicolumn{2}{|c|}{$E_{\text {con }} \log _{10} C F U / m L$} & \multicolumn{2}{|c|}{$E_{\max } \log _{10} \mathrm{CFU} / \mathrm{mL}$} & \multicolumn{2}{|c|}{$\mathrm{EC}_{50} \mathrm{fAUC} / \mathrm{MIC}$} & \multirow[t]{2}{*}{$r^{2}$} \\
\hline & Estimate & Standard error & Estimate & Standard error & Estimate & Standard error & \\
\hline 4 & 7.89 & 0.13 & 2.07 & 0.19 & 6.65 & 20.64 & 0.99 \\
\hline 7 & 8.20 & 0.29 & 2.69 & 0.50 & 154.66 & 110.15 & 0.98 \\
\hline 10 & 8.12 & 0.41 & 2.23 & 0.88 & 294.18 & 390.79 & 0.92 \\
\hline 14 & 8.33 & 0.02 & 1.32 & 0.03 & 197.34 & 16.58 & 1.00 \\
\hline 21 & 9.11 & 0.10 & 1.26 & 0.14 & 0.00 & 74.35 & 0.99 \\
\hline 26 & 9.92 & 0.84 & 1.46 & 1.16 & 0.00 & 173.21 & 0.70 \\
\hline
\end{tabular}

bacterial population became rifapentine resistant after just 26 days of the monotherapy. The difference in the total vs. drug resistant population was not statistically different $[p>$ 0.05]. Figure $4 \mathrm{D}$ show the inhibitory sigmoid $\mathrm{E}_{\max }$ curves with different rifapentine exposures for each sampling timepoint and Table 4 summarize the extent of bacterial kill and other model parameters for rifapentine performance in the HFS-MAC.

In Figures 5A-C we show the time kill curves with different rifabutin AUC/MIC exposures, as well as emergence of rifabutin resistance over 26 days study period. The overall kill, with the three rifabutin exposures, during the first 4 days of therapy was 0.9 (95\% CI: 0.61 to 1.24$) \log _{10} \mathrm{CFU} / \mathrm{mL}$ below stasis with a kill rate of $0.23 \pm 0.03 \log _{10} \mathrm{CFU} / \mathrm{mL} /$ day. Despite there was no pre-existing rifabutin resistant subpopulation in the inoculum, we noticed rapid emergence of rifabutin resistance in the HFS-MAC. The relationship between the different rifabutin exposures and bacterial burden could be explained in Figure 5D and Table 5 summarize the other model parameters as calculated in the HFSMAC. Table 6 summarize each rifamycin drug exposure, $E_{\max }$, kill slopes, and difference in the total and drug resistant MAC subpopulation as achieved in the HFS-MAC.

\section{DISCUSSION}

While there are guidelines and recommendations in place, the treatment of pulmonary MAC is not straight forward and 


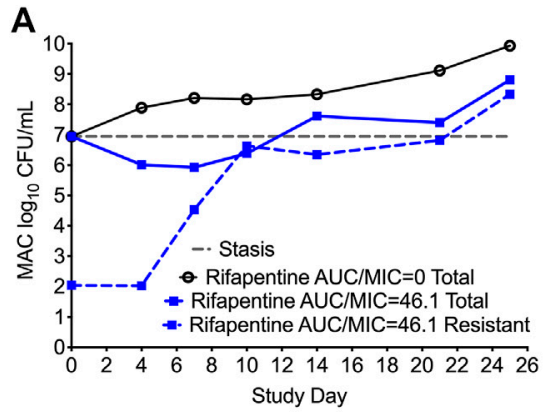

C

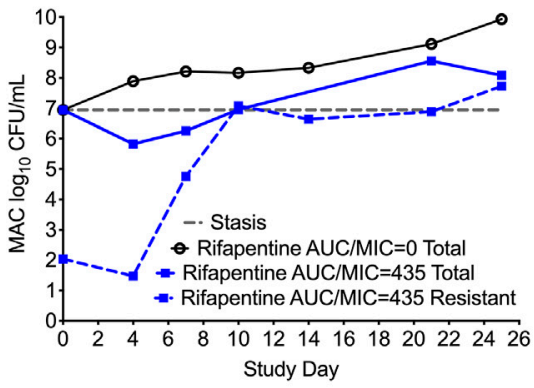

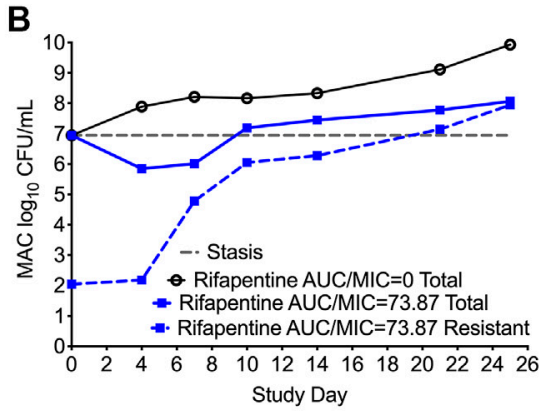

D

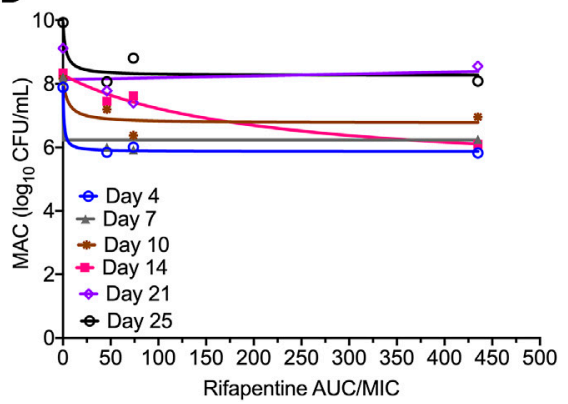

FIGURE 4 | Rifapentine kill curve and resistance emergence in the HFS-MAC. (A-C). There was no difference in the total as well as drug resistant subpopulation among the three rifapentine exposures tested in the HFS-MAC. The rifapentine monotherapy failed after 4 days of treatment. (D). Inhibitory Sigmoid $E_{\max }$ model showed highest $r^{2}=0.993$ and lowest Akaike Information Criteria score (Akaike, 1974) $=0.080$ on study day 4, where EC $\mathrm{C}_{50}$ was calculated as AUC/MIC of 1.025 .

TABLE 4 | Rifapentine pharmacokinetics/pharmacodynamics indices for microbial kill in the HFS-MAC.

\begin{tabular}{|c|c|c|c|c|c|c|c|}
\hline \multirow[t]{2}{*}{ Study day } & \multicolumn{2}{|c|}{$E_{\text {con }} \log _{10} \mathrm{CFU} / \mathrm{mL}$} & \multicolumn{2}{|c|}{$E_{\max } \log _{10} \mathrm{CFU} / \mathrm{mL}$} & \multicolumn{2}{|c|}{$\mathrm{EC}_{50}$ fAUC/MIC } & \multirow[t]{2}{*}{$r^{2}$} \\
\hline & Estimate & Standard error & Estimate & Standard error & Estimate & Standard error & \\
\hline 4 & 7.88 & 0.13 & 2.02 & 0.20 & 1.02 & 5.18 & 0.99 \\
\hline 10 & 8.16 & 0.58 & 1.40 & 0.87 & 2318.36 & 4.95 & 0.79 \\
\hline 14 & 8.28 & 0.30 & 3.14 & 1.00 & 50.39 & 0.00 & 0.96 \\
\hline 21 & 8.13 & 0.66 & 0.00 & 0.00 & 50.32 & 0.00 & 0.02 \\
\hline 26 & 9.93 & 0.59 & 1.67 & 0.88 & 0.00 & 2.89 & 0.85 \\
\hline
\end{tabular}

TABLE 5 | Rifabutin pharmacokinetics/pharmacodynamics indices for microbial kill in the HFS-MAC.

\begin{tabular}{|c|c|c|c|c|c|c|}
\hline \multirow[t]{2}{*}{ Study day } & \multicolumn{2}{|c|}{$E_{c o n} \log _{10} C F U / m L$} & \multicolumn{2}{|c|}{$E_{\max } \log _{10} \mathrm{CFU} / \mathrm{mL}$} & \multicolumn{2}{|c|}{$\mathrm{EC}_{50}$ fAUC/MIC } \\
\hline & Estimate & Standard error & Estimate & Standard error & Estimate & Standard error \\
\hline 4 & 7.88 & 0.37 & 1.68 & 0.58 & 0.00 & 171.80 \\
\hline 10 & 8.06 & 0.50 & 4.29 & 8.25 & 2318.36 & 7350.63 \\
\hline 14 & 8.33 & 0.15 & 1.21 & 0.26 & 50.39 & 125.52 \\
\hline 21 & 9.11 & 0.27 & 1.30 & 0.46 & 50.32 & 209.32 \\
\hline 26 & 9.93 & 0.98 & 0.83 & 1.53 & 0.00 & 904.01 \\
\hline
\end{tabular}

treatment failure as well as relapse is common. Moreover, in a recent study Griffith et al. (Griffith and Aksamit, 2020) argued that the "actual adherence to the guideline-based treatment approach by the clinicians is low". In the recent years there has been attempts to optimize the treatment of MAC using the principles of PK/PD (Deshpande et al., 2010a; Deshpande et al., 2010b; Deshpande and Gumbo, 2011; Deshpande et al., 2016a; Deshpande et al., 2016b; Deshpande et al., 2017a; Deshpande et al., 2017b; Deshpande et al., 2017c; Deshpande et al., 2017d; Srivastava et al., 2017a; Srivastava et al., 2017b), as summarized in 


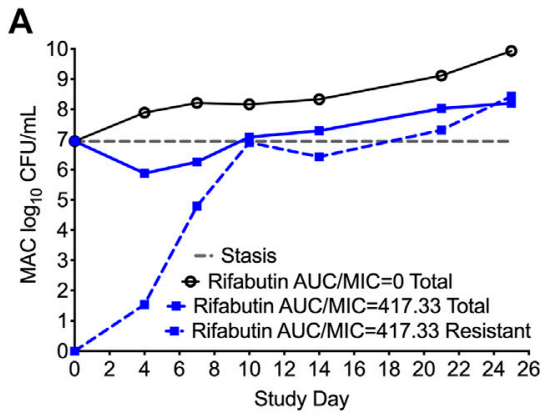

C

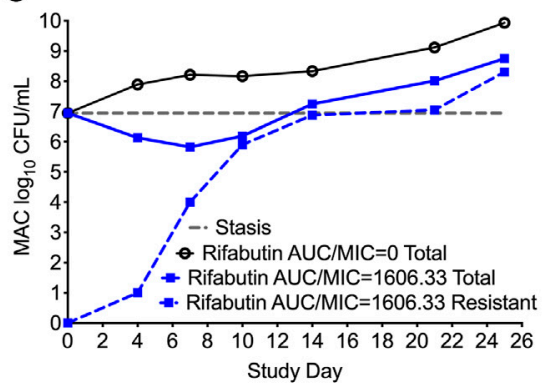

B

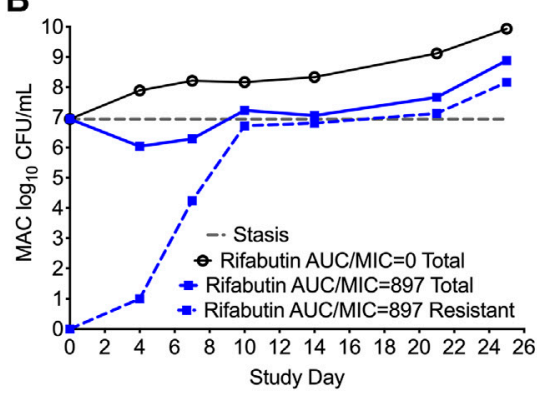

D

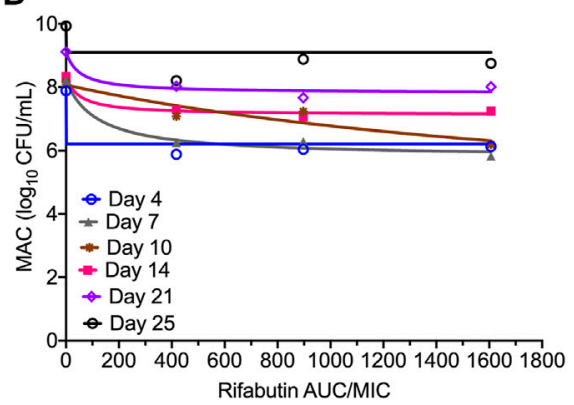

FIGURE 5 | Rifabutin monotherapy in the HFS-MAC and resistance emergence (A-C). Kill curve and emergence of rifabutin resistance with each of the three exposures tested in the HFS-MAC. Though there were no pre-existing rifabutin resistance colonies recorded in the inoculum, monotherapy led to rapid rifabutin resistance and entire MAC population was rifabutin resistant on day 25 (D). Relationship between the rifabutin exposure and bacteria burden using the inhibitory sigmoid $\mathrm{E}_{\max }$ model. The $\mathrm{EC}_{50}$ was calculated as AUC/MIC of 118.4 on day 7 of the study.

TABLE 6 | Comparison of kill slopes and bacterial burden in HFS-MAC treated with different rifamycins.

Drug exposure (fAUC/MIC)
Kill slope for day 0-4 $\left(\log _{10} \mathrm{CFU} / \mathrm{ml} / \mathrm{day}\right)$
Difference with non-treated control $\left(\log _{10} \mathrm{CFU} / \mathrm{mL}\right)$
Difference between total and drug-resistant population $\left(\log _{10} \mathrm{CFU} / \mathrm{mL}\right.$ )
Rifampin 192.97

Rifampin 633.33

Rifampin 1328

Rifapentine 46.1

Rifapentine 73.87

Rifapentine 435

Rifabutin 417.33

Rifabutin 897

Rifabutin 1606.33
Kill between day

1.07
1.00
1.19
1.10
0.93
1.12
1.06
0.90
0.81

1.07

1.00

1.19
1.10

0.93

1.12

1.06

0.81

$$
\begin{aligned}
& -0.27 \\
& -0.25 \\
& -0.30 \\
& -0.27 \\
& -0.23 \\
& -0.28 \\
& -0.27 \\
& -0.23 \\
& -0.20
\end{aligned}
$$

\subsection{6}

0.80

1.89

1.87

1.13

1.85

1.73

1.05

1.18
1.45

0.71

0.00

0.12

0.47

0.35

$-0.23^{\mathrm{a}}$

0.72

0.45

${ }^{a}$ Negative value show that the drug resistant population replaced entire drug susceptible population.

Table 7. However, the applicability of the PK/PD indices in the treatment of MAC remains to be understudied. Rifamycins are one of the recommended class of drug in the MAC combination regimen. However, pre-clinical PK/PD studies comparing rifampin, rifapentine, and rifabutin for MAC bacterial kill and resistance suppression are lacking. Such studies are important especially when new drug/drug delivery methods are being developed for MAC treatment and swapping of one rifamycin with other is recommended to prevent drug resistance (Griffith and Aksamit, 2020).

In the present study, we compared the three rifamycins mimicking human-like PK of each drug, and all three rifamycins failed to control the MAC growth despite showing initial bacterial kill, which was not significantly different from each other. The emergence of acquired drug resistance to the three rifamycins was also very similar and did not differ between the three drug exposures. We noticed that while the rifampin AUC/MIC $=192.97$ exposure failed to kill MAC, had the lowest drug resistant subpopulation compare to the high drug exposures. Among the three rifamycins, there are clinical studies reporting rifampin is well tolerated in patients at dose up to $2400 \mathrm{mg}$ /day (Zurr et al., 2016). Thus, formal dose-response studies are warranted to determine if higher rifampin exposure can overcome the resistance emergence. Further, the rifamycin's MIC of the laboratory strain used in the studies was $0.032 \mathrm{mg} / \mathrm{L}$, whereas the $\mathrm{MIC}_{90}$ for the clinical strains was substantially 
TABLE 7 | Pharmacokinetics/pharmacodynamics parameters of different drug against MAC.

\begin{tabular}{|c|c|c|c|c|c|c|c|}
\hline Drug & $\begin{array}{l}\text { PK-PD } \\
\text { index }\end{array}$ & $\mathbf{E}_{\max }$ & $\begin{array}{l}\text { Kill below } \\
\text { stasis }\end{array}$ & $\mathrm{EC}_{80}$ & $\begin{array}{l}\text { Stasis } \\
\text { exposure }\end{array}$ & $\begin{array}{c}1.0 \log _{10} \mathrm{CFU} / \mathrm{mL} \\
\text { exposure }\end{array}$ & $\begin{array}{c}2.0 \log _{10} \mathrm{CFU} / \mathrm{mL} \\
\text { exposure }\end{array}$ \\
\hline $\begin{array}{l}\text { Azithromycin Deshpande et al. } \\
\text { (2016a) }\end{array}$ & $\begin{array}{l}\mathrm{AUC}_{0-} \\
{ }_{24} / \mathrm{MIC}\end{array}$ & 2.11 & 0.6 & 3.43 & 1.29 & NA & NA \\
\hline $\begin{array}{l}\text { Ethambutol Deshpande et al. } \\
\text { (2010a) }\end{array}$ & $\mathrm{C}_{\max } / \mathrm{MIC}$ & 0.79 & NA & 1.23 & NA & NA & NA \\
\hline $\begin{array}{l}\text { Moxifloxacin Deshpande et al. } \\
\text { (2010b) }\end{array}$ & $\begin{array}{l}\mathrm{AUC}_{\mathrm{O}-} \\
{ }_{24} / \mathrm{MIC}\end{array}$ & 3.03 & 3.0 & 163.37 & 6.0 & 17.12 & 391.56 \\
\hline $\begin{array}{l}\text { Thioridazine Deshpande et al. } \\
\text { (2016b) }\end{array}$ & $\mathrm{C}_{\max } / \mathrm{MIC}$ & 7.19 & 5.38 & 0.88 & 0.35 & 0.45 & 0.5 \\
\hline Linezolid Deshpande et al. (2017b) & $\begin{array}{l}\mathrm{AUC}_{0-} \\
{ }_{24} / \mathrm{MIC}\end{array}$ & 2.1 & 1.06 & 42 & 7.82 & 40.83 & NA \\
\hline Tidezolid Deshpande et al. (2017d) & $\begin{array}{l}\mathrm{AUC}_{0} \\
{ }_{24} / \mathrm{MIC}\end{array}$ & 3.78 & 2.07 & 21.71 & 17.15 & 23.46 & 37.5 \\
\hline CAV Deshpande et al. (2017a) & $\% \mathrm{~T}_{\mathrm{MIC}}$ & 2.71 & 2.4 & 52 & 9.0 & 70 & 100 \\
\hline Tigecycline $^{\mathrm{a}}$ & $\begin{array}{l}\mathrm{AUC}_{\mathrm{O}-} \\
{ }_{24} / \mathrm{MIC}\end{array}$ & 3.87 & 4.94 & 59 & 6.0 & 10.93 & 33.74 \\
\hline
\end{tabular}

aUnpublished data; CAV, ceftazidime-avibactam; $E_{\max }$ and kill below stasis represent $\log _{10} \mathrm{CFU} / \mathrm{mL}$; stasis, initial bacterial burden; NA, Not achieved.

high. The probability of achieving optimal drug exposure decrease with increase in MIC. Thus, present results may be overoptimistic regarding rifamycin's performance in the clinical setting.

Our study has limitations. First, we show that the rifamycins failed to control the bacterial growth when tested as monotherapy. The treatment of MAC pulmonary infection is a combination of drugs where drugs may protect each other in terms of resistance emergence. However, PK/PD studies with ethambutol and azithromycin show minimal efficacy of these drugs (Deshpande et al., 2010a; Deshpande et al., 2016a). Therefore, more effective drugs need to be explored/developed for successful treatment outcome. Second, we tested only three different doses of each rifamycin for efficacy and resistance suppression in MAC. Such study design may not accurately determine the optimal drug exposure for maximal kill and resistance suppression. Third, since rifapentine and rifabutin have long half-life compare to rifampin, dose-fractionations studies are required to determine the optimal dosing schedule to achieve the maximum efficacy, assuming the high dose will not be associated with severe adverse event, in combination of other effective anti-MAC drugs. Finally, the HFS-MAC lacks the complex immune system that may also play role in therapy outcome.

In summary, rifampin, rifapentine and rifabutin have comparable efficacy against MAC in the in vitro HFS-MAC

\section{REFERENCES}

Akaike, H. (1974). A new look at the statistical model identification. IEEE Trans. Autom. Control. 19 (6), 716-723. doi:10.1109/TAC.1974.1100705

Anonymous (2014). Mycobutin [Rifabutin] capsule, USP [Online]. Available: https:// www.accessdata.fda.gov/drugsatfda_docs/label/2014/050689Orig1s018lbl.pdf [Accessed 1020 2020].

Blaschke, T. F., and Skinner, M. H. (1996). The clinical pharmacokinetics of rifabutin. Clin. Infect. Dis. 22 (Suppl. 1), S15-S22. doi:10.1093/clinids/22. supplement_1.s15 model, and all three fail to control the MAC growth despite showing early bactericidal activity. The optimal rifamycin exposure for MAC kill remains to be determined.

\section{DATA AVAILABILITY STATEMENT}

The original contributions presented in the study are included in the article/Supplementary material, further inquiries can be directed to the corresponding author.

\section{AUTHOR CONTRIBUTIONS}

Conceptualization and design, SS and TG; MIC and HFS-MAC experiments, GDB, UMJ, SS, BJ; PK/PD modeling, TG and SS. SS wrote the first draft of the manuscript. All authors reviewed and approved the final version of the manuscript.

\section{FUNDING}

SS is supported by funding from the department of Pulmonary Immunology (423500/14000), University of Texas System STARS award (250439/39411), and 1R21AI148096-01 from the National Institute of Allergy and Infectious Diseases (NIAID).

Chapagain, M., Gumbo, T., Heysell, S., and Srivastava, S. (2020). The novel regimen of rifapentine, tedizolid, and minocycline is potent and could shorten therapy duration for pulmonary Mycobacterium kansasii. Am. J. Respir. Crit. Care Med. 201, A4372. doi:10.1164/ajrccm-conference.2020. 201.1_MeetingAbstracts.A4372

Clsi (2018). Susceptibility testing of mycobacteria, nocardia spp., and other aerobic actinomycetes. 3rd ed. CLSI Standard M24. (Wayne, PA: Clinical and Laboratory Standards Institute).

Cowman, S., Van Ingen, J., Griffith, D. E., and Loebinger, M. R. (2019). Nontuberculous mycobacterial pulmonary disease. Eur. Respir. J. 54, 1900250. doi:10.1183/13993003.00250-2019 
Deshpande, D., and Gumbo, T. (2011). Pharmacokinetic/pharmacodynamic-based treatment of disseminated Mycobacterium avium. Future Microbiol. 6 (4), 433-439. doi:10.2217/fmb.11.25

Deshpande, D., Pasipanodya, J. G., and Gumbo, T. (2016a). Azithromycin dose to maximize efficacy and suppress acquired drug resistance in pulmonary Mycobacterium avium disease. Antimicrob. Agents Chemother. 60 (4), 2157-2163. doi:10.1128/aac.02854-15

Deshpande, D., Srivastava, S., Chapagain, M. L., Lee, P. S., Cirrincione, K. N., Pasipanodya, J. G., et al. (2017a). The discovery of ceftazidime/avibactam as an anti-Mycobacterium avium agent. J. Antimicrob. Chemother. 72 (suppl_2), 36-42. doi:10.1093/jac/dkx306

Deshpande, D., Srivastava, S., Meek, C., Leff, R., and Gumbo, T. (2010a). Ethambutol optimal clinical dose and susceptibility breakpoint identification by use of a novel pharmacokinetic-pharmacodynamic model of disseminated intracellular Mycobacterium avium. Antimicrob. Agents Chemother. 54 (5), 1728-1733. doi:10.1128/aac.01355-09

Deshpande, D., Srivastava, S., Meek, C., Leff, R., Hall, G. S., and Gumbo, T. (2010b). Moxifloxacin pharmacokinetics/pharmacodynamics and optimal dose and susceptibility breakpoint identification for treatment of disseminated Mycobacterium avium infection. Antimicrob. Agents Chemother. 54 (6), 2534-2539. doi:10.1128/aac.01761-09

Deshpande, D., Srivastava, S., Musuka, S., and Gumbo, T. (2016b). Thioridazine as chemotherapy for Mycobacterium avium complex diseases. Antimicrob. Agents Chemother. 60 (8), 4652-4658. doi:10.1128/aac.02985-15

Deshpande, D., Srivastava, S., Pasipanodya, J. G., and Gumbo, T. (2017b). Linezolid as treatment for pulmonary Mycobacterium avium disease. J. Antimicrob. Chemother. 72 (suppl_2), i24-i29. doi:10.1093/jac/dkx304

Deshpande, D., Srivastava, S., Pasipanodya, J. G., Lee, P. S., and Gumbo, T. (2017c). A novel ceftazidime/avibactam, rifabutin, tedizolid and moxifloxacin (CARTM) regimen for pulmonary Mycobacterium avium disease. J. Antimicrob. Chemother. 72 (suppl_2), i48-i53. doi:10.1093/jac/dkx307

Deshpande, D., Srivastava, S., Pasipanodya, J. G., Lee, P. S., and Gumbo, T. (2017d). Tedizolid is highly bactericidal in the treatment of pulmonary Mycobacterium avium complex disease. J. Antimicrob. Chemother. 72 (suppl_2), 30-35. doi:10. 1093/jac/dkx305

Dooley, K. E., Bliven-Sizemore, E. E., Weiner, M., Lu, Y., Nuermberger, E. L., Hubbard, W. C., et al. (2012). Safety and pharmacokinetics of escalating daily doses of the antituberculosis drug rifapentine in healthy volunteers. Clin. Pharmacol. Ther. 91 (5), 881-888. doi:10.1038/clpt.2011.323

Egelund, E. F., Weiner, M., Singh, R. P., Prihoda, T. J., Gelfond, J. A. L., Derendorf, H., et al. (2014). Protein binding of rifapentine and its 25-desacetyl metabolite in patients with pulmonary tuberculosis. Antimicrob. Agents Chemother. 58 (8), 4904-4910. doi:10.1128/aac.01730-13

Griffith, D. E., and Aksamit, T. R. (2020). How I Do It: managing M. avium complex lung disease with a little help from my friend. Chest. S0012-3692 (20), 34924-2. doi:10.1016/j.chest.2020.10.031

Griffith, D. E. (2007). Therapy of nontuberculous mycobacterial disease. Curr. Opin. Infect Dis. 20 (2), 198-203. doi:10.1097/qco.0b013e328055d9a2

Gumbo, T., Louie, A., Deziel, M. R., Liu, W., Parsons, L. M., Salfinger, M., et al. (2007). Concentration-dependent Mycobacterium tuberculosis killing and prevention of resistance by rifampin. Antimicrob. Agents Chemother. 51 (11), 3781-3788. doi:10.1128/aac.01533-06

Mcfarland, J. (1907). The nephelometer:an instrument for estimating the number of bacteria in suspensions used for calculating the opsonic index and for vaccines. Jama XLIX (14), 1176-1178. doi:10.1001/jama.1907.25320140022001f

Naiker, S., Connolly, C., Wiesner, L., Kellerman, T., Reddy, T., Harries, A., et al. (2014). Randomized pharmacokinetic evaluation of different rifabutin doses in African HIV-infected tuberculosis patients on lopinavir/ritonavir-based antiretroviral therapy. BMC Pharmacol. Toxicol. 15, 61. doi:10.1186/2050-6511-15-61

No_Author_Listed (2014). Mycobutin [Rifabutin] capsule, USP [Online]. Available: https://www.accessdata.fda.gov/drugsatfda_docs/label/2014/050689Orig1s018lbl. pdf [Accessed 10/20/2020 2020].

Pasipanodya, J. G., Ogbonna, D., Deshpande, D., Srivastava, S., and Gumbo, T. (2017). Meta-analyses and the evidence base for microbial outcomes in the treatment of pulmonary Mycobacterium avium-intracellulare complex disease. J. Antimicrob. Chemother. 72 (suppl_2), i3-i19. doi:10.1093/jac/dkx311

Srivastava, S., Deshpande, D., and Gumbo, T. (2017a). Failure of the azithromycin and ethambutol combination regimen in the hollow-fibre system model of pulmonary Mycobacterium avium infection is due to acquired resistance. J. Antimicrob. Chemother. 72 (suppl_2), i20-i23. doi:10.1093/jac/dkx303

Srivastava, S., Deshpande, D., Sherman, C. M., and Gumbo, T. (2017b). A “shock and awe" thioridazine and moxifloxacin combination-based regimen for pulmonary Mycobacterium avium-intracellulare complex disease. J. Antimicrob. Chemother. 72 (suppl_2), i43-i47. doi:10.1093/jac/dkx308

Srivastava, S., and Gumbo, T. (2011). In vitro and in vivo modeling of tuberculosis drugs and its impact on optimization of doses and regimens. Curr. Pharm. Des. 17 (27), 2881-2888. doi:10.2174/138161211797470192

Winthrop, K. L., Marras, T. K., Adjemian, J., Zhang, H., Wang, P., and Zhang, Q. (2020). Incidence and prevalence of nontuberculous mycobacterial lung disease in a large U.S. Managed care Health plan, 2008-2015. Ann. ATS 17 (2), 178-185. doi:10.1513/annalsats.201804-236oc

Zurr, M., Van Soolingen, D., Van Der Werf, T., Gumbo, T., and Alffenaar, J. (2016). Intermediate susceptibility dose-dependent breakpoints for high dose rifampin, isoniazid, and pyrazinamide treatment of MDR-TB in programs. Clin Infect Dis. 67 (11), 1743-1749. doi:10.1093/cid/ciy346

Conflict of Interest: TG founded Praedicare Inc., a contract research organization.

The remaining authors declare that the research was conducted in the absence of any commercial or financial relationships that could be construed as a potential conflict of interest.

Copyright (c) 2021 Boorgula, Jakkula, Gumbo, Jung and Srivastava. This is an openaccess article distributed under the terms of the Creative Commons Attribution License (CC BY). The use, distribution or reproduction in other forums is permitted, provided the original author(s) and the copyright owner(s) are credited and that the original publication in this journal is cited, in accordance with accepted academic practice. No use, distribution or reproduction is permitted which does not comply with these terms. 